\title{
FRAGILITY AND PERSISTENCE OF LEAFWISE INTERSECTIONS
}

\author{
VIKTOR L. GINZBURG AND BAŞAK Z. GÜREL
}

\begin{abstract}
In this paper we study the question of fragility and robustness of leafwise intersections of coisotropic submanifolds. Namely, we construct a closed hypersurface and a sequence of Hamiltonians $C^{0}$-converging to zero such that the hypersurface and its images have no leafwise intersections, showing that some form of the contact type condition on the hypersurface is necessary in several persistence results. In connection with recent results in continuous symplectic topology, we also show that $C^{0}$-convergence of hypersurfaces, Hamiltonian diffeomorphic to each other, does not in general force $C^{0}$-convergence of the characteristic foliations.
\end{abstract}

\section{Contents}

1. Introduction and main results

1.1. Introduction

1.2. Main results

1.3. Outline of the proofs

2. Symplectic plugs in $\mathbb{R}^{4}$

2.1. Plugs

2.2. Proofs of Theorems 1.1 and $1.3 \quad 9$

3. Proof of Proposition 2.3 12

References

\section{INTRODUCTION AND MAIN RESULTS}

1.1. Introduction. In this paper we study the question of fragility and existence of leafwise intersections of coisotropic submanifolds. Our main result is that leafwise intersections are fragile already for hypersurfaces and need not exist even for $C^{0}$-small Hamiltonians: we construct a closed hypersurface $M$ and a sequence of Hamiltonians $F_{k}, C^{0}$-converging to zero, such that $M$ and its images $\varphi_{F_{k}}(M)$ have no leafwise intersections. This shows, in particular, that some form of the contact type condition on the hypersurface $M$ is essential in Hofer's theorem, [Ho], stated below and in its generalizations. Also, in connection with the recent results from [HLS, Op], we prove that the $C^{0}$-convergence of hypersurfaces Hamiltonian

Date: September 19, 2021

2010 Mathematics Subject Classification. 53D40, 53D12, 37J45.

Key words and phrases. leafwise intersections, coisotropic submanifolds, Hamiltonian Seifert conjecture.

The work is partially supported by NSF grants DMS-1414685 (BG) and DMS-1308501 (VG). 
diffeomorphic to each other does not, in general, imply $C^{0}$-convergence of their characteristic foliations.

Let us now discuss our results in more detail and in a broader context. Consider a closed coisotropic submanifold $M$ (e.g., a hypersurface or a Lagrangian submanifold) of a symplectic manifold $W$. Let $\varphi=\varphi_{F}$ be a compactly supported Hamiltonian diffeomorphism of $W$, i.e., the time-one map of the flow generated by a time-dependent Hamiltonian $F: S^{1} \times W \rightarrow \mathbb{R}$. A leafwise intersection of $M$ and $\varphi(M)$ is a point $z \in M$, or a pair $(z, \varphi(z))$, such that $\varphi(z) \in M \cap \varphi(M)$ and moreover $z$ and $\varphi(z)$ lie on the same leaf of the characteristic foliation of $M$. Thus leafwise intersections are associated with $M$ and $\varphi$, and in the pair $(z, \varphi(z))$ it is $\varphi(z)$ that is actually in $M \cap \varphi(M)$.

To the best of the authors' knowledge, leafwise intersections were first considered in [Mo], and according to a theorem of Moser, [Mo], and Banyaga, [Ba], leafwise intersections necessarily exist when $M$ is a hypersurface and $\varphi$ is $C^{1}$-close to the identity. In fact, this is true for any closed coisotropic submanifold, as is easy to see by applying Weinstein's theorem on clean intersections, [We], to the graph of the characteristic foliation of $M$ near the diagonal; see [Mo, p. 33]. Moreover, one has the Lusternik-Schnirelmann and Morse type inequalities for the number of leafwise intersections.

Recently, Moser's theorem was strengthened by Ziltener in [Zi14], where it was shown that leafwise intersections must exist for any closed coisotropic submanifold $M$ whenever $\varphi$ is the time-one map of a Hamiltonian isotopy $\varphi^{t}$ which is $C^{0}$-close to $i d$ or, more generally, when $\varphi^{t}(M)$ stays $C^{0}$-close to $M$. Furthermore, in this case one still has the Lusternik-Schnirelmann (cup-length) and Morse type multiplicity results. This result is in some sense sharp since the condition that $\varphi$ is close to $i d$ is clearly necessary unless $M$ meets some additional requirements.

Chronologically, however, the next crucial step after Moser's theorem was a theorem of Hofer from [Ho] (see also $[\mathrm{EH}]$ ) asserting the existence of leafwise intersections for hypersurfaces in $\mathbb{R}^{2 n}$ of restricted contact type, provided that $\varphi$ has sufficiently small Hofer's norm

$$
\|\varphi\|_{H}:=\inf _{\varphi_{F}=\varphi}\|F\|_{H}, \text { where }\|F\|_{H}=\int_{S^{1}}\left(\max F_{t}-\min F_{t}\right) d t .
$$

(Here we are assuming that $\varphi$ and $F$ are compactly supported.) Moreover, in this theorem, the upper bound on $\|\varphi\|_{H}$ is given by a certain homological capacity of the domain bounded by $M$; see, e.g., [Gi07, Thm. 2.9] for a symplectic topological treatment of the question. Note also that in $\mathbb{R}^{2 n}$ leafwise intersections obviously need not exist when $\varphi$ is too far from $i d$; for in this case we can easily have $M \cap$ $\varphi(M)=\emptyset$.

Since then the problem of existence of leafwise intersections has been extensively investigated, and Hofer's theorem has been extended to coisotropic submanifolds and to other ambient symplectic manifolds; see, e.g., [AF10, AF12, AMc, AMo, Dr, Gi07, Gü, Ka, Zi09] for an admittedly incomplete but representative list of results on leafwise intersections. A common feature of these results is that, in contrast with Moser's theorem, to ensure the existence of leafwise intersections one has to impose some additional requirements on the hypersurface or the coisotropic submanifold. This is usually a variant of the contact type condition, but in [Zi09] leafwise intersections are studied under the assumption that the characteristic foliation is a fibration. 
The main result of this paper (Theorem 1.1) shows that some assumption on $M$ is indeed necessary in Hofer's theorem to guarantee the existence of leafwise intersections already when $M$ is a closed hypersurface in $\mathbb{R}^{2 n}$. It also shows that in Ziltener's theorem one cannot replace $C^{0}$-norm by Hofer's norm. To be more specific, we construct a closed smooth hypersurface $M \subset \mathbb{R}^{2 n \geq 4}, C^{0}$-close to the standard round sphere $S^{2 n-1}$, and a sequence of autonomous Hamiltonians $F_{k}$, $C^{0}$-converging to 0 and supported in the same compact set, such that $M$ and $\varphi_{F_{k}}(M)$ have no leafwise intersections for all $k$. The proof relies heavily on the construction of counterexamples to the Hamiltonian Seifert conjecture; see, e.g., [Gi99] and references therein. Note that in Theorem 1.1, the convergence to zero is much stronger than the convergence in Hofer's norm and not obviously related to the $C^{0}$-convergence of the maps $\varphi_{F_{k}}$. (Apparently, the sequence $\varphi_{F_{k}}$ we constructed does not $C^{0}$-converge; by [Zi14], it cannot $C^{0}$-converge to $i d$.)

Regarding the requirements on $M$, there is still a considerable gap between what is currently known for hypersurfaces in $\mathbb{R}^{2 n}$ and our example. For instance, it is still not known if leafwise intersections must exist when $M$ is a stable (in the sense of [HZ]) closed hypersurface in $\mathbb{R}^{2 n}$ and $\|\varphi\|_{H}$ is sufficiently small. The notion of stability can be extended to coisotropic submanifolds (see [Bo, Section 5] for the original definition and [Gi07] for a detailed discussion), and the question also makes sense for closed coisotropic submanifolds. Drawing from the results in [Us], it seems reasonable to conjecture that the right condition on $M$ for the existence of leafwise intersections of $M$ and $\varphi(M)$ when $\|\varphi\|_{H}$ is small is that the characteristic foliation of $M$ is totally geodesic with respect to some metric. However, as of this writing, this conjecture has far from been proved. Without stability (or the totally geodesic condition) it is not even known whether a closed coisotropic submanifold $M \subset \mathbb{R}^{2 n}$ with $1<\operatorname{codim} M<n$ must intersect $\varphi(M)$ when $\|\varphi\|_{H}$ is small; cf. [Gi07, Ke, Us].

Our second result concerns a different aspect of coisotropic rigidity. An important question in the area, stemming from the analogy between coisotropic and Lagrangian submanifolds, is whether or not a smooth $C^{0}$-limit $M$ of smooth coisotropic submanifolds $M_{k}$ must be coisotropic; cf. [LS] for the Lagrangian counterpart. No counterexamples are known even in the most general setting, but it is not unreasonable to impose additional requirements on $M_{k}$ of two types: stability or contact type conditions and that the submanifolds $M_{k}$ are Hamiltonian diffeomorphic to each other. Under the latter condition, one can also ask, provided that $M$ is indeed coisotropic, if the characteristic foliations of $M_{k}$ converge to the characteristic foliation of $M$. This second question is already of interest when $M_{k}$ and $M$ are hypersurfaces and hence $M$ is automatically coisotropic. (Note also that without the assumption that the hypersurfaces $M_{k}$ are symplectomorphic, the characteristic foliations need not converge, as is easy to see.) The answer to both questions is affirmative when Hamiltonian diffeomorphisms between $M_{1}$ and $M_{k}$ also $C^{0}$-converge to a homeomorphism; see [HLS] and also [BO, Op].

We show in Theorem 1.3 that without this convergence assumption or without extra assumptions on $M_{k}$ the answer to the second question is negative for hypersurfaces. Namely, our proof of Theorem 1.1 yields a sequence of hypersurfaces $M_{k} \subset \mathbb{R}^{2 n \geq 4}$ Hamiltonian diffeomorphic to each other and $C^{0}$-converging to the round sphere $S^{2 n-1} \subset \mathbb{R}^{2 n}$, but such that the characteristic foliations of $M_{k}$ do not $C^{0}$-converge to the characteristic foliation on $M=S^{2 n-1}$. More precisely, 
there exists a sequence of closed characteristics $L_{k} \subset M_{k} C^{\infty}$-converging to a simple closed curve in $S^{2 n-1}$ which is nowhere tangent to the characteristic foliation. Furthermore, the characteristic foliation of $M_{k}$ is not homeomorphic to the characteristic foliation on $S^{2 n-1}$. The hypersurfaces $M_{k}$ are diffeomorphic to $S^{2 n-1}$ but not stable; see Remark 2.6.

1.2. Main results. Before stating the main theorems of the paper, let us briefly recall relevant definitions, some of which we have already used in Section 1.1. Let $\left(W^{2 n}, \sigma\right)$ be a symplectic manifold; this is just the standard symplectic $\mathbb{R}^{2 n}$ in most of the results considered here. Given a Hamiltonian $F: S^{1} \times W \rightarrow \mathbb{R}$, which we will always assume to be compactly supported, we denote the (time-dependent) Hamiltonian flow of $F$ by $\varphi_{F}^{t}$ and the time-one map of this flow by $\varphi_{F}$. (Our sign convention for the Hamiltonian vector field $\xi_{F}$ of $F$ is $i_{\xi_{F_{t}}} \sigma=-d F_{t}$.) Throughout the paper, for the sake of simplicity, all maps and functions are assumed to be $C^{\infty}$-smooth unless explicitly stated otherwise.

Furthermore, recall from Section 1.1 that, given a coisotropic submanifold $M$ of $W$ (e.g., a hypersurface) and a Hamiltonian diffeomorphism $\varphi=\varphi_{F}$, a leafwise intersection of $M$ and $\varphi(M)$ is a point $z \in M$ such that $\varphi(z) \in M \cap \varphi(M)$ and $z$ and $\varphi(z)$ lie on the same leaf of the characteristic foliation of $M$. Here $\varphi(z)$, rather than $z$, is actually an intersection of $M$ and $\varphi(M)$. This, however, should cause no problem since $\varphi$ gives rise to a one-to-one correspondence between the leafwise intersections $z$ and the points $\varphi(z)$. Sometimes we will also refer to the pair $(z, \varphi(z))$ as a leafwise intersection. (Although the definitions of leafwise intersections vary between different papers, this one, arguably the most naive, is sufficient for our purposes.) Leafwise intersections of $M$ and $\varphi(M)$ depend on $M$ and the map $\varphi$, but only on the pair of coisotropic submanifolds $M$ and $\varphi(M)$. We refer the reader to, e.g., [Gi07] for a general discussion of coisotropic submanifolds in the context of symplectic topology.

The main result of the paper is the following.

Theorem 1.1. There exists a closed, smooth hypersurface $M \subset \mathbb{R}^{2 n}, 2 n \geq 4$, and a sequence of $C^{\infty}$-smooth autonomous Hamiltonians $F_{k} \stackrel{C^{0}}{\rightarrow} 0$, supported in the same compact set, such that $M$ and $\varphi_{F_{k}}(M)$ have no leafwise intersections.

Here the hypersurface $M$ cannot have contact type by the results of [Ho] and, in fact, $M$ is not even stable in the sense of [HZ]; see Remark 2.6 for a proof of this fact.

Remark 1.2. It readily follows from the proof that $M$ can be chosen to be diffeomorphic and arbitrarily $C^{0}$-close to the round sphere $S^{2 n-1}$, and the Hamiltonians $F_{k}$ can also be chosen to be supported in an arbitrarily small neighborhood of $S^{2 n-1}$. To be more precise, for any $\delta>0$, we can ensure that $M$ is the image of an embedding which is $\delta$-close to the standard embedding $S^{2 n-1} \hookrightarrow \mathbb{R}^{2 n}$ and that for all $k$ the Hamiltonians $F_{k}$ are supported in the $\delta$-neighborhood of $S^{2 n-1}$ and $\varphi_{F_{k}}$ is also $\delta$-close to $i d$. As has been pointed out above, $\varphi_{F_{k}}$ cannot $C^{0}$-converge to $i d$ for a fixed $M$ due to the results from [Zi14].

As a byproduct of the proof of Theorem 1.1, we obtain the following result.

Theorem 1.3. There exists a sequence of closed, smoothly embedded hypersurfaces $M_{k} \subset \mathbb{R}^{2 n}, 2 n \geq 4$, Hamiltonian diffeomorphic to each other and $C^{0}$-converging 
and diffeomorphic to the round sphere $S^{2 n-1} \subset \mathbb{R}^{2 n}$, but such that there exists a sequence of closed characteristics $L_{k} \subset M_{k} C^{\infty}$-converging to a simple closed curve in $S^{2 n-1}$ which is nowhere tangent to the characteristic foliation and intersects every characteristic at at most one point. Furthermore, the characteristic foliation on $M_{k}$ is not homeomorphic to the characteristic foliation (the Hopf fibration) on the sphere $S^{2 n-1}$.

The first assertion of the theorem should be understood as that the characteristic foliations on $M_{k}$ do not $C^{0}$-converge to the characteristic foliation on $S^{2 n-1}$. (Here we leave aside a somewhat delicate matter of defining $C^{0}$-convergence of foliations (cf. [Ep]) further complicated by the fact that in this context $M_{k}$ are different, although diffeomorphic, manifolds.) The assumption that the hypersurfaces $M_{k}$ are Hamiltonian diffeomorphic to each other is essential - without it, it is obvious that the characteristic foliations on $M_{k}$ need not to converge to the characteristic foliation on $M$ in any sense.

1.3. Outline of the proofs. The proofs of Theorems 1.1 and 1.3 rely heavily on the methods developed to construct counterexamples to the Hamiltonian Seifert conjecture; see, e.g., [Gi99].

Let $S^{2 n-1}$ be the unit sphere in $\mathbb{R}^{2 n}$ with standard Darboux coordinates, say, $\left(p_{1}, q_{1}, \ldots, p_{n}, q_{n}\right)$. Set $\tilde{F}=\epsilon \chi \cdot p_{1}$, where $\chi$ is a cut-off function equal to one near $S^{2 n-1}$. Near $S^{2 n-1}$, the map $\varphi_{\tilde{F}}$ is the parallel transport by the vector $w=(0, \epsilon, 0, \ldots, 0)$. For $\epsilon>0$ small, the only leafwise intersections of $S^{2 n-1}$ and $\varphi_{\tilde{F}}\left(S^{2 n-1}\right)$ are two points $z^{ \pm}$on the unit circle $S$ in the $\left(p_{1}, q_{1}\right)$-plane, located near the North and the South Poles on $S^{2 n-1}$. (The points $\varphi_{\tilde{F}}\left(z^{ \pm}\right)$are the intersections of $S$ and the transported circle $S+(0, \epsilon)$ in $\mathbb{R}^{2}$.) Let us now insert two symplectic plugs into $S^{2 n-1}$ to interrupt $S$ between $z^{+}$and $\varphi_{\tilde{F}}\left(z^{+}\right)$and between $z^{-}$and $\varphi_{\tilde{F}}\left(z^{-}\right)$as in, e.g., [Gi95, Gi99]; see Fig. 1. Here, however, since our goal is just to break the characteristic $S$, we can use the plugs from [Ci] with circular cores. Hence the only dimensional constraint is that $2 n \geq 4$. We choose the plugs narrow and thin, with width much smaller than $\epsilon / 2$, located in a very small neighborhood of the intersection of $S^{2 n-1}$ and the plane $q_{1}=0$, and place them in such a way that they are displaced by $\varphi_{\tilde{F}}$. As a result, we obtain a new hypersurface $M$ which is $C^{0}$-close to $S^{2 n-1}$, differs from $S^{2 n-1}$ only within the plugs, and such that the characteristic $S$ is broken into several characteristics: one containing $z^{ \pm}$and some other containing $\varphi_{\tilde{F}}\left(z^{ \pm}\right)$. This procedure is illustrated in Fig. 1 . We claim that $M$ and $\varphi_{\tilde{F}}(M)$ have no leafwise intersections. Indeed, the points $z^{ \pm}$are no longer leafwise intersections for $M$ and $\varphi_{\tilde{F}}(M)$, and since the plugs are displaced and due to the plug-symmetry conditions, no new leafwise intersections are created.

Applying this construction to a sequence $\epsilon_{k} \rightarrow 0$, we obtain a sequence of perturbations $M_{k}$ of $S^{2 n-1}$ and a sequence of Hamiltonians $\tilde{F}_{k}=\epsilon_{k} \chi \cdot p_{1}$ such that $M_{k}$ and $\varphi_{\tilde{F}_{k}}\left(M_{k}\right)$ have no leafwise intersections and $\tilde{F}_{k} \stackrel{C^{\infty}}{\rightarrow} 0$. Note also that the sequence $M_{k}$ can be chosen to $C^{0}$-converge to $S^{2 n-1}$, and this is essential for the proof of Theorem 1.3.

So far we have stayed close to the construction from [Gi07, Example 7.2]. Now a crucial new step is the observation (Proposition 2.3) that the plugs can be inserted so that all hypersurfaces $M_{k}$ are Hamiltonian diffeomorphic to $M=M_{1}$, i.e., there exists a sequence of Hamiltonian diffeomorphisms $\eta_{k}: \mathbb{R}^{2 n} \rightarrow \mathbb{R}^{2 n}$ such that $\eta_{k}(M)=M_{k}$, and the maps $\eta_{k}$ are supported within the same compact set. (The 


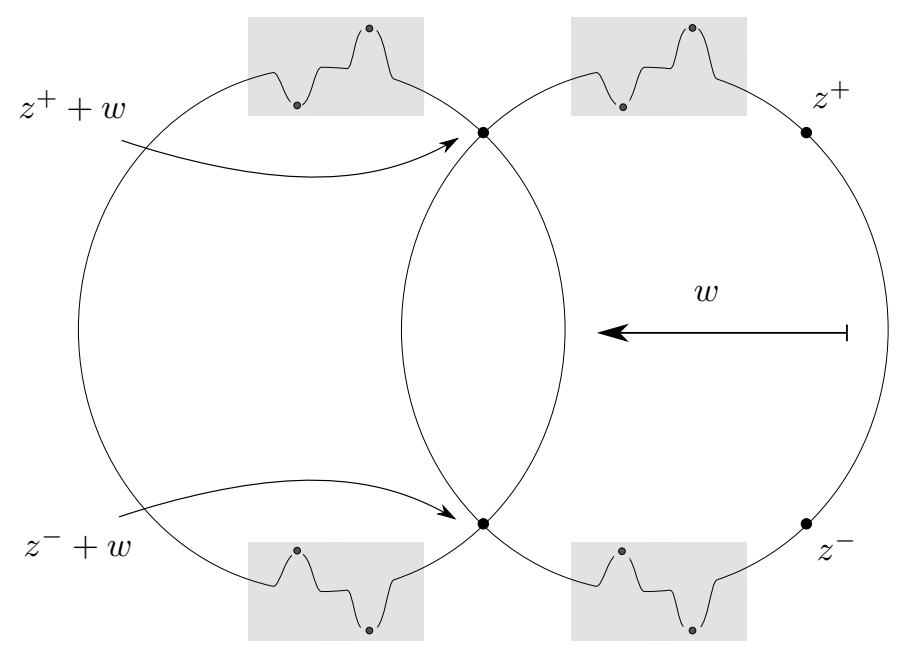

FiguRE 1. Breaking leafwise intersections.

key point is to find "arbitrarily thin" plugs Hamiltonian diffeomorphic to a given one. A Hamiltonian diffeomorphism is constructed using Moser's method, which ultimately reduces to a variant of a (singular) Cauchy problem for a first-order PDE. We solve the Cauchy problem by the standard method of characteristics, but extra care is needed at this step to account for singularities.) Then the Hamiltonians $F_{k}=\tilde{F}_{k} \circ \eta_{k}$ are also supported within the same compact set. Clearly, $\varphi_{F_{k}}(M)$ and $M$ have no leafwise intersections, and $F_{k} \stackrel{C^{0}}{\rightarrow} 0$. This proves Theorem 1.1 and also Theorem 1.3 with $M_{k}$ taken as the required sequence of hypersurfaces.

This argument is essentially independent of the dimension $2 n$, and hence here we only detail it for $2 n=4$. The general case can be handled in a similar fashion. Namely, as in other Hamiltonian plug constructions (see, e.g., [Gi99]), one takes the product of the lower-dimensional plug $P$ described here and the symplectic ball $B^{2 m}$ and equips $P \times B^{2 m}$ with a $\mathrm{U}(m)$-invariant "plug two-form" standard near the boundary of $P \times B^{2 m}$.

The proof is organized as follows. In Section 2, we describe the plugs, state Proposition 2.3, and derive Theorems 1.1 and 1.3 from the proposition. Proposition 2.3 is then proved in Section 3.

\section{SympleCtic Plugs in $\mathbb{R}^{4}$}

2.1. Plugs. In this section we discuss the construction of a symplectic plug in the setting specifically tailored to the proof of Theorem 1.1; we refer the reader to, e.g., [Gi99] for a treatment of the plugs in a much more general context. Let $\Pi=[-\delta, \delta] \times[-T, T]$, for some $\delta>0$ and $T>0$, with coordinates $(x, t)$ and let $P=S^{1} \times \Pi$. We denote the angle coordinate on $S^{1}$ by $\theta$. For two auxiliary functions $f$ and $H$ on $\Pi$ to be specified later, set

$$
\omega=d(H d \theta-f d t) .
$$


Furthermore, consider the product $B=P \times[-a, a]$ for some $a>0$, and denote by $y$ the coordinate on $[-a, a]$. Thus we have

$$
B=\underbrace{S^{1}}_{\theta} \times \underbrace{[-\delta, \delta]}_{x} \times \underbrace{[-T, T]}_{t} \times \underbrace{[-a, a]}_{y} .
$$

We equip $B$ with the symplectic form

$$
\sigma=d x \wedge d \theta+d y \wedge d t
$$

and identify $P$ with the subset $y=0$ of $B$.

Lemma 2.1. Assume that $f_{x}^{\prime}$ and $H_{x}^{\prime}$ do not vanish simultaneously, $|f|<a$ and $|H| \leq \delta$, and that $f \equiv 0$ and $H \equiv x$ near $\partial \Pi$. Then $\omega$ is a maximally non-degenerate form on $P$ with characteristic vector field

$$
X=f_{x}^{\prime} \frac{\partial}{\partial \theta}-H_{t}^{\prime} \frac{\partial}{\partial x}+H_{x}^{\prime} \frac{\partial}{\partial t}=f_{x}^{\prime} \frac{\partial}{\partial \theta}+\xi_{H},
$$

where $\xi_{H}$ is the Hamiltonian vector field of $H$ on $(\Pi, d x \wedge d t)$. In other words, $\operatorname{rk} \omega=2$ and $i_{X} \omega=0$ and $X \neq 0$. Furthermore, there exists an embedding $j: P \rightarrow$ $P \times(-a, a)$ such that $j=(i d, 0)$ near $\partial P$ and $j^{*} \sigma=\omega$.

Proof. Set

$$
j(\theta, x, t)=(\theta, H(x, t), t,-f(x, t)) .
$$

Clearly, $j=(i d, 0)$ near $\partial P$, since $f \equiv 0$ and $H \equiv x$ near $\partial \Pi$, and $j^{*} \sigma=\omega$. It readily follows from the assumption that $f_{x}^{\prime}$ and $H_{x}^{\prime}$ do not vanish simultaneously that $j$ is an embedding. Hence $j^{*} \sigma$ is maximally non-degenerate. Finally, a direct calculation shows that $i_{X} \omega=0$ and $X \neq 0$.

We require the functions $H$ and $f$ to meet the following conditions:

(P1) $H \equiv x$ and $f \equiv 0$ near $\partial \Pi$, and $|f|<a$ and $|H| \leq \delta$ on $\Pi$;

(P2) $H_{x}^{\prime} \geq 0$;

(P3) the critical points of $H$ are $p_{ \pm}=\left(0, \tau_{ \pm}\right)$and $f_{x}^{\prime}\left(p_{ \pm}\right) \neq 0$, while $H_{x}^{\prime}=0$ only at these points;

(P4) $H$ is even in $t$ and $f$ is odd in $t$ for any $x$; in particular, $\tau_{-}=-\tau_{+}$.

Note that these requirements include the conditions of Lemma 2.1. It is easy to see that such functions $H$ and $f$ do exist for any positive parameters $\delta, T$ and $a$. The level sets of $H$ and its Hamiltonian flow are shown in Fig. 2.

Lemma 2.2. Assume that the functions $H$ and $f$ satisfy $(P 1)-(P 4)$. Then $X=$ $\partial / \partial t$ near $\partial P$. The (local) flow of $X$ has exactly two integral curves entirely contained in $P$ : these are the periodic orbits $S^{1} \times\left\{p_{ \pm}\right\}$. For every integral curve which both enters and exits $P$ (i.e., meets the parts of $\partial P$ where $t= \pm T$ ), the exit and entrance points have the same $x$ and $\theta$ coordinates. There exist "trapped" integral curves, i.e., the integral curves that enter but do not exit the plug.

Proof. The first two assertions and also the last assertion readily follow from the explicit expression for $X$ given by (2.2). The third assertion is a straightforward consequence of the fact that, by $(\mathrm{P} 4)$, the reflection in the $\{t=0\}$-plane changes the sign of the $(\theta, x)$-component of $X$; see, e.g., [Gi99, Sect. 2.2] for more details.

When the functions $H$ and $f$ meet conditions (P1)-(P4), we will refer to $P$ equipped with the form $\omega$ defined by (2.1) together with the embedding $j$ given by (2.3) as a symplectic plug or, when the role of the parameters $(\delta, T, a)$ and/or of 


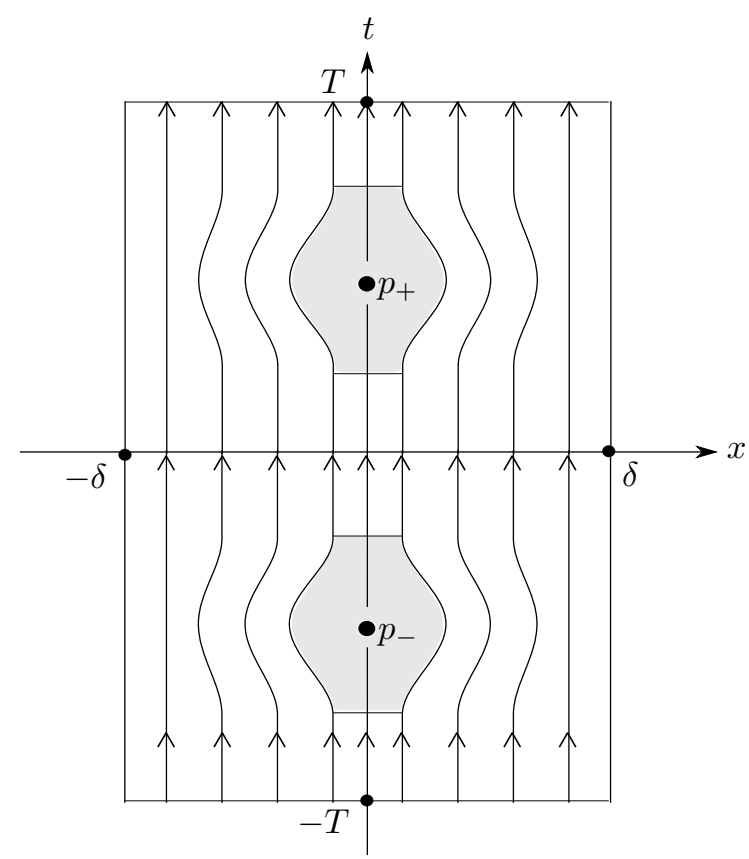

Figure 2. The rectangle $\Pi$, the levels of $H$ and the critical points $p_{ \pm}=\left(0, \tau_{ \pm}\right)$, and the neighborhoods $W$ (shaded) from the proof of Proposition 2.3.

the functions $H$ and $f$ is essential, as the $(\delta, T, a ; H, f)$-plug or just the $(H, f)$-plug. The key to the proof of Theorem 1.1 is

Proposition 2.3. For any $\delta>0$ there exists a sequence of $\left(\delta, T_{k}, a_{k} ; H_{k}, f_{k}\right)$-plugs such that $T_{k} \rightarrow 0$ and $a_{k} \rightarrow 0$, and the plugs are Hamiltonian diffeomorphic with diffeomorphisms equal to id near $\partial B$.

Remark 2.4. The last assertion of the proposition might, perhaps, require a clarification. Without loss of generality we may assume that the sequences $T_{k} \rightarrow 0$ and $a_{k} \rightarrow 0$ are strictly monotone decreasing. Set

$$
\Pi_{k}=[\delta, \delta] \times\left[-T_{k}, T_{k}\right], P_{k}=S^{1} \times \Pi_{k} \text { and } B_{k}=P_{k} \times\left[-a_{k}, a_{k}\right] .
$$

These are sequences of nested sets. Therefore, we can also view a sequence of $\left(\delta, T_{k}, a_{k} ; H_{k}, f_{k}\right)$-plugs as defined on the same set $P=P_{1}$ and $B=B_{1}$, but with

$$
\operatorname{supp}\left(H_{k}-x\right) \subset \Pi_{k} \text { and }\left\|f_{k}\right\|_{C^{0}}<a_{k} .
$$

Furthermore, denote by $j_{k}$ the embedding $j$ of $P_{k}$ into $B_{k}$ given by (2.3). Likewise, we can interpret $j_{k}$ as a map from $P$ to $B$ by extending it from $P_{k}$ to $P$ as $(i d, 0)$. Then the proposition asserts that, for a suitably chosen sequence of plugs, there exists a sequence of Hamiltonian diffeomorphisms $\eta_{k}: B \rightarrow B$ equal to $i d$ near $\partial B$ and sending the image of $j$ to the image of $j_{k}$. (Note that $\eta_{k}$ is not required, in any sense, to conjugate the maps $j$ and $j_{k}$.)

Remark 2.5. For our purposes it would be sufficient to have such a sequence of plugs with only one parameter, say $a$, going zero, while the other one, $T$, remaining 
fixed. However, we find the (superficially) stronger version of the proposition stated above more intuitive. Also, the stronger version comes essentially for free as an easy consequence of its one-parameter counterpart: to make $T$ and $a$ both small, rather than just $a$, it suffices to apply a (suitably cut-off) hyperbolic transformation in the $(t, y)$-plane; see the proof of the proposition in Section 3.

2.2. Proofs of Theorems 1.1 and 1.3. Assuming Proposition 2.3 and postponing its proof to the next section, let us now prove Theorems 1.1 and 1.3.

Proof of Theorem 1.1. The argument follows the line of reasoning outlined in Section 1.3. Set $2 n=4$ and let $S^{3}$ be the unit sphere in $\mathbb{R}^{4}$ with Darboux coordinates $\left(p_{1}, q_{1}, p_{2}, q_{2}\right)$. Consider the parallel transport along the $q_{1}$-axis in small $\epsilon>0$, i.e., the map

$$
\left(p_{1}, q_{1}, p_{2}, q_{2}\right) \mapsto\left(p_{1}, q_{1}, p_{2}, q_{2}\right)+w, \text { where } w=(0, \epsilon, 0,0) .
$$

After cutting off outside a neighborhood of $S^{3}$, we can view this map as the Hamiltonian diffeomorphism $\varphi_{\tilde{F}}$ generated by the Hamiltonian $\tilde{F}=\epsilon \chi \cdot p_{1}$, where $\chi$ is a cut-off function equal to one near $S^{3}$, on a shell which contains both $S^{3}$ and $S^{3}+w$.

For $\epsilon>0$ small, the only leafwise intersections of $S^{3}$ and $\varphi_{\tilde{F}}\left(S^{3}\right)$ are the two points $z^{ \pm}$on the unit circle $S$ in the $\left(p_{1}, q_{1}\right)$-plane, located near the North Pole, $(1,0,0,0)$, and the South Pole, $(-1,0,0,0)$, of $S^{3}$ and such that

$$
\left\{\varphi_{\tilde{F}}\left(z^{+}\right), \varphi_{\tilde{F}}\left(z^{-}\right)\right\}=S \cap(S+w) .
$$

More precisely, $z^{ \pm}=\left( \pm \sqrt{1-\epsilon^{2} / 4},-\epsilon / 2,0,0\right)$.

To see that there are no other leafwise intersections, note first that $z$ is a leafwise intersection if and only if $z$ and $\varphi_{\tilde{F}}(z)=z+w$ lie on the same Hopf circle. This Hopf circle is then the unit circle in the real 2-plane spanned by $z$ and $z+w$, obviously containing $w$. But this plane must also be a complex line in $\mathbb{R}^{4}=\mathbb{C}^{2}$, and hence it must also contain $i w$. This forces the plane to be the $\left(p_{1}, q_{1}\right)$-coordinate plane and $z$ to be one of the points $z^{ \pm}$.

Fix now an embedding $\psi$ (or $\psi^{+}$) of $S^{1}$ into a small neighborhood of the North Pole in the intersection of $S^{3}$ with the hyperplane $q_{1}=0$, sending $\theta=0$ to the North Pole. For a sufficiently small $\delta>0$, we can extend this embedding to a symplectic embedding $\Psi$ (or $\Psi^{+}$) of $S^{1} \times[-\delta, \delta]$, equipped with the symplectic form $\sigma=d x \wedge d \theta$, into $S^{3} \cap\left\{q_{1}=0\right\}$. Such an extension exists because $S^{3} \cap\left\{q_{1}=0\right\}$ is symplectic near the North Pole. (Note also that $\Psi$ does not pass through $z^{+}$, since $z^{+}$lies in the $q_{1}=-\epsilon / 2$ plane.)

Furthermore, let $U=U^{+}$be a neighborhood of $Y=\Psi\left(S^{1} \times[-\delta, \delta]\right)$ in $\mathbb{R}^{4}$. We assume that $U$ is so small that the following conditions are met:

- $z^{+} \notin U$;

- $\varphi_{\tilde{F}}^{\tau}(U)$ is contained in the region where $\chi \equiv 1$ for all $\tau \in[0,1]$;

- $U$ is displaced by $\varphi_{\tilde{F}}$, i.e., $U \cap(U+w)=\emptyset$;

- $U$ does not intersect the shifted sphere $\varphi_{\tilde{F}}\left(S^{3}\right)=S^{3}+w$.

The last two conditions require $U$ to be "narrow and low".

When the parameters $T$ and $a$ of the plug are small, we can symplectically embed $B=P \times[-a, a]$ into $U$ extending the embedding $\Psi$ of $S^{1} \times[-\delta, \delta] \times\{0\} \times\{0\}$ to $B$ and sending $P$ into $V=U \cap S^{3}$. Since the embedding is symplectic, it sends the characteristics in $P$ to the characteristics in $V \subset S^{3}$. In particular, the $t$-axis in $P$ (i.e., the line $x=0=\theta$ ) matches the characteristic through the North Pole 
(or, equivalently, through $z^{+}$) in $S^{3}$. From now on, we identify $P$ and $B$ with their images under this embedding.

Such an embedding does exist because $\Psi$ is symplectic. To be more precise, due to this assumption and a variant of the symplectic neighborhood theorem, we may without loss of generality assume that $U$ has the form

$$
U=S^{1} \times\left(-\delta^{\prime}, \delta^{\prime}\right) \times\left(-T^{\prime}, T^{\prime}\right) \times\left(-a^{\prime}, a^{\prime}\right)
$$

with coordinates $\left(\theta, x^{\prime}, t^{\prime}, y^{\prime}\right)$ and the symplectic form

$$
\sigma^{\prime}=d x^{\prime} \wedge d \theta+d y^{\prime} \wedge d t^{\prime}
$$

and that $V \subset U$ is given by the condition $y^{\prime}=0$ and the North Pole is the origin $(0,0,0,0)$ in these coordinates. Furthermore, we can also assume that $\Psi(\theta, x)=$ $(\theta, x, 0,0)$. (Hence, $\delta<\delta^{\prime}$.) Now the required embedding, pulling back $\sigma^{\prime}$ to $\sigma$, is

$$
(\theta, x, t, y) \mapsto\left(\theta, x^{\prime}, t^{\prime}, y^{\prime}\right)=(\theta, x, \kappa t, y / \kappa)
$$

where $\kappa$ is fixed and the positive parameters $T$ and $a$ are chosen so small that and $\kappa T<T^{\prime}$ and $a / \kappa<a^{\prime}$. (In fact, since we are free to chose any $\kappa$, it would be sufficient to vary only one of the parameters $a$ and $T$. For instance, having $T$ fixed, we can take $\kappa=T^{\prime} / 2 T$ and then $a$ so small that $a / \kappa=2 a T / T^{\prime}<a^{\prime}$.)

It is essential for what follows that in this construction $\delta^{\prime}$, and hence $\delta$, can be taken independent of $\epsilon$, while $T^{\prime}$ and $a^{\prime}$ (and thus $T$ and $a$ or at least one of these parameters) are bounded from above by some functions of $\epsilon$.

Next, we repeat this process starting with embeddings $\psi^{-}$of $S^{1}$ and $\Psi^{-}$of $S^{1} \times[-\delta, \delta]$ into a neighborhood of the South Pole in $S^{3} \cap\left\{q_{1}=0\right\}$ and passing through the South Pole. As a result, we have an embedding of $B$ into a small neighborhood $U^{-}$of the band $Y^{-}=\Psi^{-}\left(S^{1} \times[-\delta, \delta]\right)$ in $\mathbb{R}^{4}$, which sends $P$ into $V^{-}=U^{-} \cap S^{3}$.

Replacing $P^{ \pm}=P$ by $Q^{ \pm}=j(P)$ in both neighborhoods $U^{ \pm}$, we obtain a new hypersurface $M$ which differs from $S^{3}$ only within $U^{ \pm}$and is $C^{0}$-close to $S^{3}$; see Fig. 1. We claim that there are no leafwise intersections of $M$ and $\varphi_{\tilde{F}}(M)$.

To prove this, we need to show that for any characteristic $\mathcal{C}$ on $M$, the sets $\mathcal{C}$ and $\varphi_{\tilde{F}}(\mathcal{C})=\mathcal{C}+w$ do not intersect. Observe that $U^{ \pm}$have been chosen to be so small that the sets $\varphi_{\tilde{F}}\left(U^{ \pm}\right)=U^{ \pm}+w$ do not intersect $M$. In particular, by our choice of $U^{ \pm}$, we have $M \cap(M+w)=S^{3} \cap\left(S^{3}+w\right)$, which is the intersection of $S^{3}$ with the hyperplane $q_{1}=\epsilon / 2$. Therefore, all leafwise intersections on $M$ must be outside $U^{ \pm}$. Every characteristic $\mathcal{C}$ on $M$ comprises (possibly) some arcs of a Hopf circle and (possibly) characteristics in $Q^{ \pm}$. We emphasize that, by Lemma 2.2, the arcs lie on the same Hopf circle. As a consequence, a leafwise intersection $z \in \mathcal{C}$ can only be located on an arc of a Hopf circle and must also be a leafwise intersection for $S^{3}$. Hence $z=z^{ \pm}$. These two points lie on the same characteristic $\mathcal{C}_{0}$ on $M$. This characteristic is the union of an arc of the unit circle in the $\left(p_{1}, q_{1}\right)$-plane contained in the $q_{1}>0$ half-plane and two characteristics in $Q^{ \pm}$, which are "trapped", i.e., enter $Q^{ \pm}$but never leave. Clearly, $\mathcal{C}_{0}$ does not intersect $\varphi_{\tilde{F}}\left(\mathcal{C}_{0}\right)=\mathcal{C}_{0}+w$, and hence $z^{ \pm}$are not leafwise intersections of $M$ with $\varphi_{\tilde{F}}(M)$.

Let us now consider a sequence $\epsilon_{k} \rightarrow 0$, set $\tilde{F}_{k}=\epsilon_{k} \chi \cdot p_{1}$, and carry out the above construction for every $k$. Namely, with embeddings $\Psi^{ \pm}$fixed (independent of $k$ ), we can, as above, take a sequence of smaller and smaller neighborhoods $U_{k}^{ \pm}$of the bands $Y^{ \pm}$and a nested sequence of symplectic embeddings of $B_{k}=P_{k} \times\left[-a_{k}, a_{k}\right]$ into $U_{k}^{ \pm}$with $T_{k} \rightarrow 0$ and $a_{k} \rightarrow 0$. We again identify $B_{k}$ and $P_{k}$ with their images 
$B_{k}^{ \pm}$and, respectively, $P_{k}^{ \pm}$in $U_{k}^{ \pm}$. Denote by $Q_{k}^{ \pm}$the images of $j_{k}\left(P_{k}\right)$ in $B_{k}^{ \pm}$and by $M_{k}$ the hypersurface obtained from $S^{3}$ by replacing $P_{k}^{ \pm}$by $Q_{k}^{ \pm}$. By construction, $M_{k}$ and $\varphi_{\tilde{F}_{k}}\left(M_{k}\right)$ have no leafwise intersections.

By Proposition 2.3 and Remark 2.4, this can be done so that there exist Hamiltonian diffeomorphisms $\eta_{k}^{ \pm}$of $B_{1}^{ \pm}$, equal to $i d$ near the boundary and sending $Q_{1}^{ \pm}$ to $Q_{k}^{ \pm} \cup\left(P_{1}^{ \pm} \backslash P_{k}^{ \pm}\right)$. We extend $\eta_{k}^{ \pm}$to a Hamiltonian diffeomorphism $\eta_{k}$ of $\mathbb{R}^{4}$ as the identity map outside $B_{1}^{ \pm}$. Setting $M=M_{1}$, we have $\eta_{k}(M)=M_{k}$. Let $F_{k}=\tilde{F}_{k} \circ \eta_{k}$. Then $M$ and $\varphi_{F_{k}}(M)$ have no leafwise intersections. Furthermore, $\left\|F_{k}\right\|_{C^{0}}=\epsilon_{k} \rightarrow 0$ and $\operatorname{supp} F_{k}=\operatorname{supp} \chi$. Thus we can ensure that $\operatorname{supp} F_{k}$ is contained in an arbitrarily small (but fixed, i.e., independent of $k$ ) neighborhood of $S^{3}$ and that $M$ is $C^{0}$-close to $S^{3}$.

Remark 2.6 (Stability). The hypersurface $M$ constructed in the proof of Theorem 1.1 is not stable in the sense of [HZ]. Indeed, one of the equivalent definitions of stability is that there exists a one-form $\alpha$ on $M$ non-vanishing on the characteristic foliation and such that $\operatorname{ker} \omega \subset \operatorname{ker} d \alpha$, where $\omega$ is the restriction of the ambient symplectic form to $M$; see, e.g., [Gi07, EKP] for a discussion of stability. Then it suffices to show that the plug $(P, \omega)$ with $\omega$ given by $(2.1)$ is not stable in the sense of this definition. Arguing by contradiction, assume that such a form $\alpha$ exists. The curves $\gamma_{ \pm}=S^{1} \times\left\{p_{ \pm}\right\}$form the boundary of the cylinder

$$
\Sigma=S^{1} \times\{0\} \times\left[\tau_{-}, \tau_{+}\right] \subset P
$$

foliated by the characteristics of $\omega$. Hence, due to the condition $\operatorname{ker} \omega \subset \operatorname{ker} d \alpha$, we have $\left.d \alpha\right|_{\Sigma}=0$. Therefore, by Stokes' theorem,

$$
\int_{\gamma_{-}} \alpha=\int_{\gamma_{+}} \alpha
$$

where $\gamma_{ \pm}$are oriented by fixing an orientation of $S^{1}$. On the other hand, the direction of the vector field $X$ defined by (2.2) matches this orientation on one of the curves $\gamma_{ \pm}$and the opposite orientation on the other. Thus, since $\alpha(X) \neq 0$ everywhere, these two integrals are non-zero and have opposite signs. Note that, as a consequence, none of the hypersurfaces $M_{k}$ is stable. Furthermore, the characteristic foliation of $M_{k}$ (or $M$ ) is not totally geodesic since for hypersurfaces this requirement is equivalent to stability; see [Us].

Proof of Theorem 1.3. It is clear that the sequence of hypersurfaces $M_{k}$ constructed in the proof of Theorem $1.1 C^{0}$-converges to the round sphere $S^{3}$. The characteristic foliation of $M_{k}$ has non-compact leaves, e.g., the characteristics which enter and remain trapped in the plugs. Thus this foliation is not homeomorphic to the characteristic foliation on $S^{3}$ since the latter is just the Hopf fibration. Finally, working, say, in a neighborhood of the North Pole, we can take one of the two characteristics $j_{k}\left(S^{1} \times p_{ \pm}\right)$as $L_{k}$, where $p_{ \pm}$are the critical points of $H$. These characteristics $C^{\infty}$-converge to the embedded circle $\Psi\left(S^{1} \times\{0\}\right) \subset S^{3}$ which is clearly nowhere tangent to the Hopf fibration and intersects every fiber at at most one point.

Remark 2.7. When $2 n \geq 6$, we could have also used, with only minor modifications to the proof, more elaborate plugs from the constructions of counterexamples to the Hamiltonian Seifert conjecture; see, e.g., [Gi99] and references therein. This would result in hypersurfaces $M$ without closed characteristics and leafwise intersections. 


\section{Proof of Proposition 2.3}

Throughout the proof, we fix positive parameters $\delta, T$ and $a$. It is sufficient to find a sequence of functions $H_{k}$ and $f_{k}$ satisfying (P1)-(P4) and such that

(i) $\left\|f_{k}\right\|_{C^{0}} \rightarrow 0$ and

(ii) $\operatorname{supp}\left(H_{k}-x\right) \subset[\delta, \delta] \times\left[-T_{k}, T_{k}\right]$ for some sequence $T_{k} \rightarrow 0$, and that the resulting plugs are Hamiltonian diffeomorphic. The last requirement means that, in the notation from Section 2, there exists a sequence of Hamiltonian diffeomorphisms of $B$ equal to $i d$ near $\partial B$ and sending $Q=j_{1}(P)$ to $Q_{k}=j_{k}(P)$; see Remark 2.4.

We start by fixing a function $H$ and considering a family of functions $f=f_{s}(x, t)$, depending smoothly on $s \in[0,1]$, and meeting conditions (P1)-(P4) for every $s$. Moreover, we require (P1) to hold uniformly in $s$, i.e., that $f_{s} \equiv 0$ for all $s$ on some neighborhood of $\partial \Pi$ independent of $s$. Furthermore, we assume that

(F) $f_{s}(x, t)=f_{0}(x, t)+c(s)$ near the points $p_{ \pm}=\left(0, \tau_{ \pm}\right)$, where $c(s)$ is a function of $s$ only.

This condition plays a crucial role in the proof. Note that here again, (F) is required to hold uniformly in $s$, i.e., each of the points $p_{ \pm}$has a neighborhood independent of $s$ where $f_{s}=f_{0}+c(s)$. (In what follows, we will always require conditions of this type to hold uniformly, without mentioning this specifically again.)

We will show that the resulting family of plugs is Hamiltonian diffeomorphic. This is clearly enough to construct a sequence of Hamiltonian diffeomorphic plugs satisfying (i) and having $H$ fixed. Then, as was pointed out in Remark 2.5, it is easy to have (ii) also satisfied by applying a cut-off hyperbolic transformation in the $(t, y)$-plane. We will carry out the argument in detail at the end of the proof.

Denote by $j_{s}$ the embedding $P \rightarrow B$ given by (2.3) for $\left(H, f_{s}\right)$, and set

$$
\omega_{s}=j_{s}^{*} \sigma=d\left(H d \theta-f_{s} d t\right) .
$$

The key step of the proof is the following result showing that these forms are diffeomorphic to each other:

Lemma 3.1. There exists an isotopy $\psi_{s}, s \in[0,1]$, of $P$ equal to id on a neighborhood of $\partial P$ and such that $\psi_{s}^{*} \omega_{s}=\omega_{0}$.

Proof. We use Moser's homotopy method. It suffices to find an $s$-dependent vector field $Z_{s}$ generating $\psi_{s}$. As is easy to see, $Z_{s}$ then must satisfy the equation

$$
L_{Z_{s}} \omega_{s}=d\left(\frac{\partial f_{s}}{\partial s}\right) \wedge d t
$$

We look for a solution $Z_{s}$ of the form

$$
Z_{s}=g_{s} \frac{\partial}{\partial \theta}
$$

where $g_{s}$ is an (unknown) function on $\Pi=[-\delta, \delta] \times[-T, T]$ depending on the parameter $s \in[0,1]$ and vanishing near $\partial \Pi$.

Calculating the right and the left hand sides of (3.1) explicitly, we arrive at the equation

where

$$
\left(\left(g_{s}\right)_{t}^{\prime} H_{x}^{\prime}-\left(g_{s}\right)_{x}^{\prime} H_{t}^{\prime}\right) d x \wedge d t=F_{s} d x \wedge d t
$$

$$
F_{s}=\frac{\partial}{\partial s}\left(\frac{\partial f_{s}}{\partial x}\right) .
$$


Equivalently, this equation can be rewritten as

$$
L_{\xi_{H}} g_{s}=F_{s},
$$

where $\xi_{H}$ is the Hamiltonian vector field of $H$ on $\Pi$. We need to find a solution $g_{s}$ of (3.2) which vanishes on a neighborhood of $\partial \Pi$ for all $s$. The right hand side $F_{s}$ of (3.2) has the following properties:

- $F_{s}$ is odd in $t$ for every $s$ and $x$ (by (P4));

- $F_{s}$ vanishes for all $s$ on some neighborhoods of the zeros $p_{ \pm}$of $\xi_{H}$ (by $(\mathrm{F})$ );

- $F_{s}$ vanishes for all $s$ on a neighborhood of the boundary ӘП.

These are the only features of $F_{s}$ needed for the proof. (In contrast, a rather specific form of $\xi_{H}$ determined by (P1)-(P4) is essential.)

We will solve equation (3.2) using the method of characteristics; see, e.g, [Ar, Chap. 2]. A somewhat non-obvious point is then the existence and smoothness of a solution near the zeros $p_{ \pm}$of $\xi_{H}$. Thus, before turning to the task of solving the equation, let us state and prove the existence and smoothness criterion we will use. (We are assuming below that $H$ is $C^{\infty}$-smooth.)

Let $W$ be a small neighborhood of one of the points $p_{ \pm}$. For the sake of simplicity, let us also assume that $W$ is cut out by two horizontal lines $t=$ const at the top and the bottom and by two levels of $H$ on its sides; see Fig. 2 and Fig. 3. In particular, the intersection of $W$ with every integral curve of $\xi_{H}$ is connected. Furthermore, assume that $g$ is a function on the slit neighborhood $\stackrel{\circ}{W}=W \backslash\left(\{0\} \times\left\{t \geq \tau_{ \pm}\right\}\right)$ constant along every integral curve of $\xi_{H}$ in this set. (We think of $g$ as a possibly non-smooth solution of the homogeneous equation $L_{\xi_{H}} g=0$ in $W$.) Finally, let us require the restriction of $g$ to the cross section $\Gamma=W \cap\left\{t=t_{0}\right\}$ to be $C^{k}$-smooth, where $t_{0}<\tau_{ \pm}$is close to $\tau_{ \pm}$; see Fig. 3. (Thus $\Gamma$ intersects all levels of $H$ in $W$.) Then we claim that $g$ extends to a $C^{k}$-smooth function on $W$. Such an extension is automatically unique and constant along the levels of $H$ in $W$. In particular, $g$ can be thought of as a true solution of the homogeneous equation in $W$.

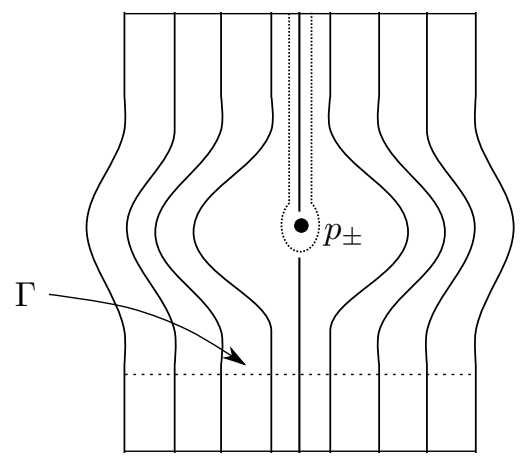

Figure 3. Neighborhood $W$, the slit $\{0\} \times\left\{t \geq \tau_{ \pm}\right\}$and the cross section $\Gamma$.

Let us prove this claim. By definition, the value of the extension at a point $z \in W$ is equal to the value of $g$ at the point $z^{\prime}$ where the level of $H$ through $z$ intersects $\Gamma$. (A continuous extension, if it exists, must have this property. Note also that, since we only need to extend $g$ to the slit $\{0\} \times\left\{t \geq \tau_{ \pm}\right\}$, we could have just set 
$g \equiv g\left(0, t_{0}\right)$ on the cut.) To see that the extension defined in this way is $C^{k}$-smooth, we observe first that, by (P2) and (P3), $H_{x}^{\prime}>0$ along $\Gamma$. Thus the restriction of $H$ to $\Gamma$ is a diffeomorphism onto its image $H(\Gamma)=H(W)$. Let us denote by $\left(\left.H\right|_{\Gamma}\right)^{\text {inv }}$ the inverse diffeomorphism. This map is as smooth as $H$, and hence $C^{\infty}$-smooth. Now it is easy to see that the extension is given by $\left.g\right|_{\Gamma} \circ\left(\left.H\right|_{\Gamma}\right)^{\text {inv }} \circ H$ on $W$. Indeed, this composition is equal to $g$ on $\stackrel{\circ}{W}$. Furthermore, every function in the composition is at least $C^{k}$-smooth. Hence the extension is also $C^{k}$-smooth on $W$.

Of course, there is also an obvious parametric version of this result. Namely, now the function $g$ depends on a parameter $s \in[0,1]$ and $\left.g\right|_{\Gamma}$ is $C^{k}$ in $x$ and $s$. Then $g$ is $C^{k}$ on $W \times[0,1]$.

With these observations in mind we are ready to solve (3.2). We do this in several steps. For the sake of simplicity, we suppress the parameter $s$ in the notation.

We set the "initial condition" $g \equiv 0$ on the bottom part $\{t=-T\}$ of $\partial \Pi$ in the Cauchy problem (3.2). The method of characteristics guarantees the existence of a smooth solution $g$ on the cut domain $\Pi \backslash\left(\{0\} \times\left[\tau_{-}, T\right]\right)$; see, e.g., [Ar, Chap. 2]. By the above criterion, since $F_{s} \equiv 0$ near $p_{-}$, the solution $g$ extends to the union of the cut domain with a neighborhood of $p_{-}$. Applying the method of characteristics again with the initial condition on the horizontal line just above $p_{-}$, we can extend $g$ to the domain $\Pi \backslash\left(\{0\} \times\left[\tau_{+}, T\right]\right)$. Next, again, by the smoothness criterion, the solution extends to a neighborhood of $p_{+}$and then by the method of characteristics to the entire domain $\Pi$.

Since $\xi_{H}=\partial / \partial t$ and $F \equiv 0$ near $\partial \Pi$, it is clear that the resulting solution $g$ vanishes (for all $s$ ) near $\partial \Pi$ except possibly the upper part $\{t=T\}$ of the boundary. Recall, however, that $H$ is even in $t$ by $(\mathrm{P} 4)$ and $F$ is odd in $t$. It readily follows then that the solution $g$ is even in $t$, and hence it also vanishes near the upper part of the boundary.

Our next step is to show that there exists an $s$-dependent Hamiltonian $K_{s}$ on $B$ with $j_{s}(P)=\varphi_{K}^{s}(j(P))$. In fact, we will find $K_{s}$ such that

$$
\left.\varphi_{K}^{s}\right|_{j(P)}=j_{s} \psi_{s}: P \rightarrow B
$$

In addition, $K_{s}$ is required to vanish uniformly in $s$ near $\partial B$.

Consider the vector field

$$
v_{s}=\frac{\partial}{\partial s} j_{s} \psi_{s}
$$

along the embedding $j_{s} \psi_{s}$. Then (3.3) is satisfied if and only if

$$
d K_{s}=-i_{v_{s}} \sigma
$$

along $j_{s} \psi_{s}$, i.e., at every point of $\left.T B\right|_{j_{s} \psi_{s}(P)}$. It is not very difficult to show that $K_{s}$ with this property exists if and only if the form

$$
\beta_{s}=\left(j_{s} \psi_{s}\right)^{*} i_{v_{s}} \sigma
$$

is exact on $P$.

Let us calculate this form explicitly. Consider a primitive $\lambda$ of $\sigma$ on $B$, e.g., we can take $\lambda=x d \theta+y d t$. Then extending $v_{s}$ to an $s$-dependent vector field on the entire domain $B$, we have

$$
\beta_{s}=\left(j_{s} \psi_{s}\right)^{*} L_{v_{s}} \lambda-\left(j_{s} \psi_{s}\right)^{*} d\left(\lambda\left(v_{s}\right)\right) .
$$


Hence it suffices to show that the form

$$
\alpha_{s}=\left(j_{s} \psi_{s}\right)^{*} L_{v_{s}} \lambda=\frac{d}{d s}\left(j_{s} \psi_{s}\right)^{*} \lambda
$$

is exact on $P$. By Lemma 3.1, $\psi_{s}^{*} j_{s}^{*} d \lambda$ is independent of $s$, and hence $\alpha_{s}$ is closed for all $s$. To see that this form is exact it suffices to show that its integral over any circle $S^{1} \times\{(x, t)\}$ is zero. But $\alpha_{s} \equiv 0$ near $\partial P$ because $j_{s}=(0, i d)$ and $\psi_{s}=i d$ near $\partial P$. Thus the integral is zero when $(x, t)$ is near $\partial P$ (and therefore for any circle) and the form is exact.

Finally, to have $K_{s}$ vanishing near $\partial B$ (for all $s$ ) it suffices to show that $\beta_{s}$ has a primitive vanishing near $\partial P$. We have already seen that $\alpha_{s}$, the first term in (3.4), has such a primitive since it vanishes near $\partial P$. A primitive of the second term in (3.4) is $\left(j_{s} \psi_{s}\right)^{*} \lambda\left(v_{s}\right)$. This function is also identically zero near $\partial P$ because $j_{s}=(0, i d)$ and $\psi_{s}=i d$, and hence $v_{s} \equiv 0$, near the boundary.

Now, let $(H, f)$ be a pair of functions meeting conditions (P1)-(P4). As is easy to see, we can always find a function $\tilde{f}$ with arbitrarily small $C^{0}$-norm on $\Pi$ and a family $f_{s}$ connecting $f_{0}=f$ and $f_{1}=\tilde{f}$, satisfying condition $(\mathrm{F})$ and such that the pair $\left(H, f_{s}\right)$ also meets the plug requirements (P1)-(P4) for all $s$ (uniformly in $s$ ). It follows that $(H, \tilde{f})$-plug is Hamiltonian diffeomorphic to the original $(H, f)$-plug.

In other words, given an $(H, f)$-plug, we can find an $(H, \tilde{f})$-plug, Hamiltonian diffeomorphic to the original plug, with arbitrarily $C^{0}$-small $\tilde{f}$. To finish the proof of Proposition 2.3, we need to find an $(\hat{H}, \hat{f})$-plug with both $\|\hat{f}\|_{C^{0}}$ and the $t$ component of $\operatorname{supp}(\hat{H}-x)$ arbitrarily small, which is Hamiltonian diffeomorphic to the $(H, f)$-plug.

We have $\|\tilde{f}\|_{C^{0}} \leq \epsilon$, where $\epsilon>0$ can be assumed to be arbitrarily small, and

$$
\operatorname{supp}(H-x) \subset P^{\prime}=S^{1} \times \Pi^{\prime}, \text { where } \Pi^{\prime}=\left[-\delta^{\prime}, \delta^{\prime}\right] \times\left[-T^{\prime}, T^{\prime}\right]
$$

for some positive $\delta^{\prime}<\delta$ and $T^{\prime}<T$. Furthermore, set $B^{\prime}=P^{\prime} \times\left[-a^{\prime}, a^{\prime}\right]$ with $\epsilon<a^{\prime}<a$, and consider the Hamiltonian

$$
G=-\kappa \cdot y t \cdot b(x, t, y)
$$

with $\kappa>0$ and the cut-off function $b$ is equal to one on $B^{\prime}$ and zero near $\partial B$. The Hamiltonian vector field $\xi_{G}$ is simply the hyperbolic vector field $(-\kappa t, \kappa y)$ in the $(t, y)$-plane as long as $(\theta, x, t, y) \in B^{\prime}$. (Our sign convention for the Hamilton equation is $i_{\xi_{G}} \sigma=-d G$.) Furthermore, as is easy to see, the flow of $G$ preserves the subset $P=\{y=0\} \subset B$.

Assume now that $e^{\kappa} \epsilon<a^{\prime}$ and denote by $\tilde{j}$ the embedding $j$, given by (2.3), for the $(H, \tilde{f})$-plug. Then $\varphi_{G}$ sends the image of $\tilde{j}$ to the image of $\hat{j}$, the $(\hat{H}, \hat{f})$-plug embedding, with

$$
\hat{H}(x, t)=H\left(x, e^{\kappa} t\right)
$$

and

$$
\hat{f}(x, t)=e^{\kappa} f\left(x, e^{\kappa} t\right) .
$$

In these formulas we treat $(x, t)$ as a point in $\mathbb{R}^{2}$ rather than in $\Pi$. However, $\hat{H}$ and $\hat{f}$ still satisfy $(\mathrm{P} 1)-(\mathrm{P} 4)$ with the same $\delta, T$ and $a$ as $(H, f)$ and $(H, \tilde{f})$, and hence give rise to a plug with the same sets $P$ and $B$ as the $(H, \tilde{f})$-plug and the $(H, f)$-plug. Furthermore,

$$
\|\hat{f}\|_{C^{0}}<e^{\kappa} \epsilon \text { and } \operatorname{supp}(\hat{H}-x) \subset S^{1} \times[-\delta, \delta] \times\left[-e^{-\kappa} T, e^{-\kappa} T\right] .
$$


VIKTOR GINZBURG AND BAŞAK GÜREL

The only constraint on $\kappa>0$ and $\epsilon>0$ is that $e^{\kappa} \epsilon<a^{\prime}$. Thus, by choosing a sufficiently large $\kappa$ and then a sufficiently small positive $\epsilon$, we can make $e^{\kappa} \epsilon$ and $e^{-\kappa} T$ arbitrarily small. By construction, the $(H, f)$-plug, the $(H, \tilde{f})$-plug, and the $(\hat{H}, \hat{f})$-plug are Hamiltonian diffeomorphic. It follows that starting with an arbitrary $(H, f)$-plug one can find a sequence of $\left(H_{k}, f_{k}\right)$-plugs (with $H_{1}=H$ and $\left.f_{1}=f\right)$, Hamiltonian diffeomorphic to each other and satisfying (i) and (ii). This completes the proof of Proposition 2.3.

\section{REFERENCES}

[AF10] P. Albers, U. Frauenfelder, Leaf-wise intersections and Rabinowitz Floer homology, $J$. Topol. Anal., 2 (2010), 77-98.

[AF12] P. Albers, U. Frauenfelder, Infinitely many leaf-wise intersection points on cotangent bundles, Expo. Math., 30 (2012), 168-181.

[AMc] P. Albers, M. McLean, Non-displaceable contact embeddings and infinitely many leafwise intersections, J. Symplectic Geom., 9 (2011), 271-284.

[AMo] P. Albers, A. Momin, Cup-length estimates for leaf-wise intersections, Math. Proc. Cambridge Philos. Soc., 149 (2010), 539-551.

[Ar] V.I. Arnold, Geometrical Methods in the Theory of Ordinary Differential Equations, Springer-Verlag, New York, 1988.

[Ba] A. Banyaga, On fixed points of symplectic maps, Invent. Math., 56 (1980), 215-229.

[Bo] P. Bolle, A contact condition for p-dimensional submanifolds of a symplectic manifold $(2 \leq p \leq n)$, Math. Z., 227 (1998), 211-230.

[BO] L. Buhovsky, E. Opshtein, Some quantitative results in $C^{0}$ symplectic geometry, Preprint 2014, arXiv:1404.0875.

[Ci] K. Cieliebak, Symplectic boundaries: creating and destroying closed characteristics, Geom. Funct. Anal., 7 (1997), 269-321.

[Dr] D. Dragnev, Symplectic rigidity, symplectic fixed points and global perturbations of Hamiltonian systems, Comm. Pure Appl. Math. 61 (2008), 346-370.

[EH] I. Ekeland, H. Hofer, Two symplectic fixed-point theorems with applications to Hamiltonian dynamics, J. Math. Pures Appl. 68 (1989), 467-489.

[EKP] Y. Eliashberg, S.S. Kim, L. Polterovich, Geometry of contact transformations and domains: orderability versus squeezing, Geom. Topol., textbf10 (2006), 1635-1747.

[Ep] D.B.A. Epstein, A topology for the space of foliations, in Geometry and topology (Proc. III Latin Amer. School of Math., Inst. Mat. Pura Aplicada CNPq, Rio de Janeiro, 1976), pp. 132-150. Lecture Notes in Math., Vol. 597, Springer, Berlin, 1977.

[Gi95] V.L. Ginzburg, An embedding $S^{2 n-1} \rightarrow \mathbb{R}^{2 n}, 2 n-1 \geq 7$, whose Hamiltonian flow has no periodic trajectories, IMRN, 1995, no. 2, 83-98.

[Gi99] V.L. Ginzburg, Hamiltonian dynamical systems without periodic orbits, in Northern California Symplectic Geometry Seminar, 35-48, Amer. Math. Soc. Transl. Ser. 2, vol. 196, Amer. Math. Soc., Providence, RI, 1999.

[Gi07] V.L. Ginzburg, Coisotropic intersections, Duke Math. J., 140 (2007), 111-163.

[Gü] B.Z. Gürel, Leafwise coisotropic intersections, Int. Math. Res. Not. IMRN, 2010, no. 5, 914-931.

[Ho] H. Hofer, On the topological properties of symplectic maps, Proc. Roy. Soc. Edinburgh Sect. A, 115 (1990), 25-38.

[HZ] H. Hofer, E. Zehnder, Symplectic Invariants and Hamiltonian Dynamics, Birkäuser, 1994.

[HLS] V. Humilière, R. Leclercq, S. Seyfaddini, Coisotropic rigidity and $C^{0}$-symplectic geometry, Preprint 2013, arXiv:1305.1287.

[Ka] J. Kang, Existence of leafwise intersection points in the unrestricted case, Israel $J$. Math., 190 (2012), 111-134.

[Ke] E. Kerman, Displacement energy of coisotropic submanifolds and Hofer's geometry, $J$. Mod. Dyn. 2 (2008), no. 3, 471-497.

[LS] F. Laudenbach, J.-C. Sikorav, Hamiltonian disjunction and limits of Lagrangian submanifolds, Internat. Math. Res. Notices (1994), no. 4, 161-168.

[Mo] J. Moser, A fixed point theorem in symplectic geometry, Acta Math., 141 (1978), 17-34. 
[Op] E. Opshtein, $C^{0}$-rigidity of characteristics in symplectic geometry, Ann. Sci. Éc. Norm. Supér., 42 (2009), 857-864.

[Us] M. Usher, Boundary depth in Floer theory and its applications to Hamiltonian dynamics and coisotropic submanifolds, Israel J. Math., 184 (2011), 1-57.

[We] A. Weinstein, Lagrangian submanifolds and hamiltonian systems, Ann. of Math. (2), 98 (1973), 377-410

[Zi09] F. Ziltener, Coisotropic submanifolds, leafwise fixed points, and presymplectic embeddings, J. Sympl. Geom., 7 (2009), 357-376.

[Zi14] F. Ziltener, Leafwise fixed points for $C^{0}$-small Hamiltonian flows and local coisotropic Floer homology, Preprint arXiv:1408.4578.

BG: Department of Mathematics, University of Central Florida, Orlando, FL 32816, USA

E-mail address: basak.gurel@ucf.edu

VG: Department of Mathematics, UC Santa Cruz, Santa Cruz, CA 95064, USA

E-mail address: ginzburg@ucsc.edu 